\title{
LARGE AMPLITUDE VIBRATIONS AND DAMAGE DETECTION OF RECTANGULAR PLATES
}

\author{
Emil Manoach ${ }^{1}$ and Irina Trendafilova ${ }^{2}$ \\ ${ }^{1}$ Institute of Mechanics, Bulgarian Academy of Sciences, \\ Acad. G. Bonchev Street, Bl. 4, 1113 Sofia, Bulgaria \\ e.manoach@imbm.bas.bg \\ Phone +359-2-9733140; fax: +359-2-8707498 \\ ${ }^{2}$ Department of Mechanical Engineering, University of Strathclyde \\ 75 Montrose Street, Glasgow, G1 1XJ, UK \\ Irina.Trendafilova@strath.ac.uk
}

Keywords: Plates, damage detection, large amplitude vibrations, Poincaré maps

\begin{abstract}
In this work, geometrically nonlinear vibrations of fully clamped rectangular plates are used to study the sensitivity of some nonlinear vibration response parameters to the presence of damage. The geometrically nonlinear version of the Mindlin plate theory is used to model the plate behaviour. Damage is represented as a stiffness reduction in a small area of the plate. The plate is subjected to harmonic loading with a frequency of excitation close to the first natural frequency leading to large amplitude vibrations. The plate vibration response is obtained by a pseudo-load mode superposition method. The main results are focussed on establishing the influence of damage on the vibration response of the plate and the change in the time-history diagrams and the Poincaré maps caused by the damage. Finally, a criterion and a damage index for
\end{abstract}


detecting the presence and the location of the damage is proposed. The criterion is based on analyzing the points in the Poincaré sections of the damaged and healthy plate. Numerical results for large amplitude vibrations of damaged and healthy rectangular and square plates are presented and the proposed damage index for the considered cases is calculated. The criterion demonstrates quite good abilities to detect and localise damage. 


\section{INTRODUCTION}

Vibration-based structural health monitoring (VSHM) methods are based on the fact that any changes introduced in a structure result in changes in its dynamic behaviour. Thus introduction of even a small defect will change the physical characteristics of a structure (its mass, stiffness, damping characteristics), which in turn will affect its vibration response and change its dynamic characteristics. VSHM methods are especially attractive because they are global monitoring methods in the sense that no a priori information for the location of the damage is needed and/or immediate access to the damaged part is not required. These features are especially important when the objects of monitoring are large and/or complex structures and when some parts of these structures are either inaccessible or very difficult for taking measurements.

Most of the previous efforts of researchers in a big part of the literature on VSHM are directed towards modal-based methods [1-7]. One of the main problems with these methods comes from the fact that in general damage is a local phenomenon and does not necessarily affect the global lower frequency response of the structure, which is normally measured during vibration tests. This is the reason why many modal-based methods suffer lack of sensitivity to damage when applied to different structures $[8,9]$. Another problem with a number of VSHM methods is that they rely on a certain model of the structure. As the theoretical model itself can only approximate the actual behaviour of the vibrating structure, it will introduce computational errors [3, 4]. If some nonlinearities or environmental conditions are not taken into account in the model, the methods might give a false alarm due to a discrepancy between the measured and the modelled response. 
To address some of the above mentioned problems a new paradigm in vibration-based monitoring has been emerging recently - the employment of the measured time series response of the structure for VSHM purposes. Techniques, which apply pure time series analysis will not necessarily suffer the above limitations and may provide a broader utility due to their generic approach. Most of the studies in this field are devoted to the problem of features extraction from the structural vibration response, which can be indicative for the presence of damage and its location $[9,13,14]$. In [8] the authors use the beating phenomenon for the damage detection (DD) purposes. In $[9,13]$ the authors introduce two new attractor-based metrics as damage sensitive features. The results are promising but the procedure has certain requirements for the excitation and for the experimental equipment. Other non-modal-based methods suggest the use of statistical approaches [14-16], neural networks [17], wavelet techniques [18, 19] and other generic techniques [20].

Time series analysis, which draws most of its applications from statistical analysis and nonlinear dynamics, can provide a number of features and techniques that may hold significant promise for VSHM. Although the approach seems to hold a lot of potential, there is limited research addressing VSHM methods based on nonlinear time series analysis [10-13].

In this work phase-space variables are used for damage detection purposes. The geometrically nonlinear theory of vibrations of a rectangular plate is used to study the sensitivity of some parameters of the nonlinear vibration response to the presence of damage. The plates are subjected to a harmonic loading leading to large amplitude vibrations and the influence of the damage on the time-history diagrams of the plate, as 
well as on the geometry of the phase-space is studied. Finally, a new criterion based on the Poincaré map of the plate response is proposed.

\section{BASIC EQUATIONS}

The object of the investigation is a rectangular plate with sides $a$ and $b$ and thickness $h$, subjected to a dynamic loading $p(x, y, t)$ perpendicular to the plate (Fig. 1 a). The presence of a defect can be modelled as a reduction of the plate thickness or a stiffness reduction and therefore a variation of the flexural rigidity in the governing equations is used. The basic equations of the plate motion are described below.

\subsection{Geometrical relationships}

The strain and curvature-displacements relationships associated with the mid-plane of the plate for large displacements and shear can be expressed as:

$$
\begin{aligned}
& \varepsilon_{x}^{0}=\frac{\partial u}{\partial x}+\frac{1}{2}\left(\frac{\partial w}{\partial x}\right)^{2}, \varepsilon_{y}^{0}=\frac{\partial v}{\partial y}+\frac{1}{2}\left(\frac{\partial w}{\partial y}\right)^{2}, \varepsilon_{x y}^{0}=\frac{\partial u}{\partial y}+\frac{\partial v}{\partial x}+\frac{\partial w}{\partial x} \frac{\partial w}{\partial y} \\
& \varepsilon_{x z}^{0}=\psi_{x}+\frac{\partial w}{\partial x}, \varepsilon_{y z}^{0}=\psi_{y}+\frac{\partial w}{\partial y}, k_{x}^{0}=\frac{\partial \psi_{x}}{\partial x}, k_{y}^{0}=\frac{\partial \psi_{y}}{\partial y}, k_{x y}^{0}=\frac{\partial \psi_{x}}{\partial y}+\frac{\partial \psi_{y}}{\partial x}
\end{aligned}
$$

and the strain vector is given by:

$$
\boldsymbol{\varepsilon}=\left\{\varepsilon_{x}^{0}+z k_{x}^{0}, \varepsilon_{y}^{0}+z k_{y}^{0}, \varepsilon_{x y}^{0}+z k_{x y}^{0}, f(z) \varepsilon_{x z}^{0}, f(z) \varepsilon_{y z}^{0}\right\}^{T}
$$

where $f(z)$ is a function describing the distribution of the shear strain along the plate thickness, $u(x, y, t)$ and $v(x, y, t)$ are the in-plane displacements, $w(x, y, t)$ is the transverse

displacement and $\psi_{x}(x, y, t), \quad \psi_{y}(x, y, t)$ are the angles of the rotation of the normal of the cross section to the plate mid-plane (see Fig. 1 b.).

\subsection{Constitutive equations}


Assuming that the material of the plate is linear elastic and isotropic the relations for the generalized stress and strain components are given by:

$$
\begin{aligned}
& N_{x}=A\left(\varepsilon_{x}^{0}+v \varepsilon_{y}^{0}\right), N_{y}=A\left(\varepsilon_{y}^{0}+v \varepsilon_{x}^{0}\right), N_{x y}=\frac{1-v}{2} A \varepsilon_{x y}^{0} \\
& M_{x}=D\left(\kappa_{x}^{o}+v \kappa_{y}^{o}\right), M_{y}=D\left(\kappa_{y}^{0}+v \kappa_{x}^{0}\right), \quad M_{x y}=\frac{1}{2}(1-v) D \kappa_{x y}^{0}, \\
& Q_{x}=\frac{1}{2}(1-v) k^{2} A \varepsilon_{x z}^{0}, \quad Q_{y}=\frac{1}{2}(1-v) k^{2} A \varepsilon_{y z}^{0} \\
& A(x, y)=\frac{E(x, y) h(x, y)}{1-v^{2}}, \quad D(x, y)=\frac{A(x, y) h(x, y)^{2}}{12}
\end{aligned}
$$

where $E$ is the Young modulus, $v$ is the Poison ratio, $k^{2}$ is a shear correction factor which is assumed equal to 5/6 throughout the paper [25]. In Eqns. (3) $N_{x}, N_{y}$ and $N_{x y}$ are the stress resultants in the mid-plane of the plate, $M_{x}, M_{y}$ and $M_{x y}$ are the stress couples and $Q_{x}$ and $Q_{y}$ are the transverse shear stress resultants.

\subsection{Equations of motion}

The equilibrium equations may be deduced by considering the translational equilibrium in the $x, y$ and $z$ directions and the rotational equilibrium about $x$ and $y$ which are as follows:

$$
\begin{aligned}
& \frac{\partial N_{x}}{\partial x}+\frac{\partial N_{x y}}{\partial y}+\rho h \ddot{u}_{x}=0 \\
& \frac{\partial N_{y}}{\partial y}+\frac{\partial N_{x y}}{\partial x}+\rho h \ddot{u}_{y}=0 \\
& \frac{\partial M_{x}}{\partial x}+\frac{\partial M_{x y}}{\partial y}-Q_{x}+c_{2} \frac{\partial \psi_{x}}{\partial t}+\frac{\rho h^{3}}{12} \ddot{\psi}_{x}=0 \\
& \frac{\partial M_{y}}{\partial y}+\frac{\partial M_{x y}}{\partial x}-Q_{y}+c_{2} \frac{\partial \psi_{y}}{\partial t}+\frac{\rho h^{3}}{12} \ddot{\psi}_{y}=0 \\
& \frac{\partial Q_{x}}{\partial x}+\frac{\partial Q_{y}}{\partial y}+N_{x} \frac{\partial^{2} w}{\partial x^{2}}+N_{y} \frac{\partial^{2} w}{\partial y^{2}}+2 N_{x y} \frac{\partial^{2} w}{\partial x \partial y}+c_{1} \frac{\partial w}{\partial t}+\rho h \ddot{w}=-p
\end{aligned}
$$


Here and throughout in the paper dots over variables represents the derivative with respect to the time, $c_{1}$ and $c_{2}$ denote the damping coefficients, and $\rho$ is the density of the plate material.

\subsection{Boundary and initial conditions}

In the present work fully clamped plates, i.e. plates for which all their four edges are clamped and in-plane fixed, are considered. This means that all displacements $u, v$ and $w$ and angular rotations $\psi_{x}$ and $\psi_{y}$ are zero along the boundaries.

The initial conditions are accepted in the following general form:

$$
\begin{aligned}
& w(x, y, 0)=w^{0}(x, y), \dot{w}(x, y, 0)=\dot{w}^{0}(x, y), \\
& \psi_{x}(x, y, 0)=\psi_{x}^{0}(x, y), \dot{\psi}_{y}(x, y, 0)=\dot{\psi}_{y}^{0}(x, y,), x \in[0, a], y \in[0, b]
\end{aligned}
$$

\section{SOLUTION OF THE PROBLEM}

\subsection{Reorganizing the equations of the plate motion}

Making the widely accepted assumption that mid-plane inertia effects are negligible, i.e. $\rho h \ddot{u}_{x}=\rho h \ddot{u}_{y}=0$ and moving the nonlinear terms to the right hand side, the equations of motion (5) are written in the following form:

$$
\begin{aligned}
& \frac{\partial}{\partial x}\left[A\left(\frac{\partial u}{\partial x}+v \frac{\partial v}{\partial y}\right)\right]+\frac{\partial}{\partial y}\left[\frac{(1-v) A}{2}\left(\frac{\partial u}{\partial y}+\frac{\partial v}{\partial x}\right)\right]=G^{u} \\
& \frac{\partial}{\partial y}\left[A\left(\frac{\partial v}{\partial y}+v \frac{\partial u}{\partial x}\right)\right]+\frac{\partial}{\partial x}\left[\frac{A(1-v)}{2}\left(\frac{\partial v}{\partial x}+\frac{\partial u}{\partial y}\right)\right]=G^{v}
\end{aligned}
$$




$$
\begin{aligned}
& D\left(\frac{\partial^{2} \psi_{x}}{\partial x^{2}}+v \frac{\partial^{2} \psi_{y}}{\partial x \partial y}\right)+\frac{(1-v) D}{2}\left(\frac{\partial^{2} \psi_{x}}{\partial y^{2}}+v \frac{\partial^{2} \psi_{y}}{\partial x \partial y}\right)-\frac{\left(1-v^{2}\right) k^{2} A}{2}\left(\psi_{x}+\frac{\partial w}{\partial x}\right)+c_{2} \dot{\psi}_{x}+\frac{\rho h^{3}}{12} \ddot{\psi}_{x}=0 \\
& D\left(\frac{\partial^{2} \psi_{y}}{\partial y^{2}}+v \frac{\partial^{2} \psi_{x}}{\partial x \partial y}\right)+\frac{(1-v) D}{2}\left(\frac{\partial^{2} \psi_{y}}{\partial x^{2}}+v \frac{\partial^{2} \psi_{x}}{\partial x \partial y}\right)-\frac{\left(1-v^{2}\right) k^{2} A}{2}\left(\psi_{y}+\frac{\partial w}{\partial y}\right)+c_{2} \dot{\psi}_{y}+\frac{\rho h^{3}}{12} \ddot{\psi}_{y}=0 \\
& \frac{(1-v) k A}{2}\left(\frac{\partial \psi_{x}}{\partial x}+\frac{\partial^{2} w}{\partial x^{2}}+\frac{\partial \psi_{y}}{\partial y}+\frac{\partial^{2} w}{\partial y^{2}}\right)+c_{1} \frac{\partial w}{\partial t}+\rho h \ddot{w}=-p+G_{L}^{3}
\end{aligned}
$$

where

$$
\begin{aligned}
& G^{u}=-0.5 \frac{\partial}{\partial x}\left\{A\left[\left(\frac{\partial w}{\partial x}\right)^{2}+\left(\frac{\partial w}{\partial y}\right)^{2}\right]\right\}-0.5 \frac{\partial}{\partial y}\left\{A(1-v)\left(\frac{\partial w}{\partial x} \frac{\partial w}{\partial y}\right)\right\} \\
& G^{v}=-0.5 \frac{\partial}{\partial y}\left\{A\left[\left(\frac{\partial w}{\partial x}\right)^{2}+\left(\frac{\partial w}{\partial y}\right)^{2}\right]\right\}-0.5 \frac{\partial}{\partial x}\left\{A(1-v)\left(\frac{\partial w}{\partial x} \frac{\partial w}{\partial y}\right)\right\}
\end{aligned}
$$

and $G_{L}^{3}$, denotes the component of the vector $\mathbf{G}_{L}\left(0,0, G_{L}^{3}\right)$ which is called "nonlinear force vector due to finite displacements"( see [21, 22].). $G_{L}^{3}$ has the form:

$$
G_{L}^{3}(x, y, t)=-\left(N_{x} \frac{\partial^{2} w}{\partial x^{2}}+N_{y} \frac{\partial^{2} w}{\partial x^{2}}+2 N_{x y} \frac{\partial^{2} w}{\partial x \partial y}\right)
$$

\subsection{Numerical approach}

Assuming $G^{u}$ and $G^{v}$ are known functions, Eqns. (7 a-b) form a linear system of PDEs which can be solved numerically. The solution is obtained using a finite a difference method by applying a central differences formula (see Appendix).

The left hand sides of Eqns. (7 c-e) contain only linear terms and therefore the mode superposition method can be used for their solution. 
Thus, the generalized displacements vector $\mathbf{U}=\left\{\alpha \psi_{x}, \alpha \psi_{y}, w\right\}^{T}\left(\alpha=h^{2} / 12\right)$ is expanded as a sum of the product of the vectors of the pseudo-normal modes $\mathbf{U}_{\mathrm{n}}$ and the time dependent functions $q_{\mathrm{n}}(t)$ as follows:

$\mathbf{U}=\sum_{n=1}^{N_{f}} \mathbf{U}_{n}(x, y) q_{n}(t)$.

Substituting Eqn. (10) into Eqns. (7 c-e), multiplying by $\mathbf{U}_{\mathrm{m}}(\mathrm{x}, \mathrm{y})$, integrating the product over the plate surface, invoking the orthogonallity condition, and assuming "proportional damping" in the sense $\iint\left(c_{2} \psi_{x n}^{2}+c_{2} \psi_{y n}^{2}+c_{1} w_{n}^{2}\right) d x d y=2 \xi_{n} \omega_{n}$, the equations for $q_{n}(t)$ will be "uncoupled" in the form:

$\ddot{q}_{n}(t)+2 \xi_{n} \omega_{n} \dot{q}_{n}+\omega_{n}^{2} q_{n}(t)=F_{n}(t)$

where $\omega_{n}$ are the natural frequencies of the linear elastic (undamped) Mindlin plate, $\xi_{n}$ are the modal damping parameters and

$F_{n}(t)=\iint \mathbf{U}_{n}^{T}\left[\mathbf{P}(x, y, t)+\mathbf{G}_{L}(x, y, t)\right] d x d y$,

$\mathbf{P}(r, t)=(0,0,-p)^{T}$

The initial conditions defined by Eqns (6) are transformed also in terms of $q_{n}(0)$ and $\dot{q}_{n}(0):$

$q_{n}(0)=q_{n}^{0}, \quad \dot{q}_{n}(0)=\dot{q}_{n}^{0}$,

$q_{n}^{0}=\iint\left(w^{0} w_{n}+\alpha \psi_{x}^{0} \psi_{x n}+\alpha \psi_{y}^{0} \psi_{y n}\right) d x d y, \dot{q}_{n}^{0}=\iint\left(\dot{w}^{0} \dot{w}_{n}+\alpha \dot{\psi}_{x}^{0} \dot{\psi}_{x n}+\alpha \dot{\psi}_{y}^{0} \dot{\psi}_{y n}\right) d x d y$

Using the methodology developed by Kukreti and Issa [21] the pseudo-load vector $\{\mathbf{P}+\mathbf{G}\}$ is interpolated by a quadratic time dependent polynomial, i.e.

$\mathbf{P}(x, y, \tau)+\mathbf{G}(x, y, \tau)=\mathbf{A}(x, y)+\mathbf{B}(x, y) \tau+\mathbf{C}(x, y) \tau^{2}, \quad 0 \leq \tau \leq L_{t}$ 
Where $L_{t}=t_{i+1}-t_{i} \quad$ represents the time increment, and $\tau$ which is defined as $\tau=t-t_{i}$, identifies a new time origin for each time increment.

Denoting

$\mathbf{P}_{0}(x, y)=\mathbf{P}(x, y, 0), \mathbf{P}_{1}(x, y)=\mathbf{P}\left(x, y, m L_{t}\right), \mathbf{P}_{2}(x, y)=\mathbf{P}\left(x, y, L_{t}\right)$,

$\mathbf{G}_{0}(x, y)=\mathbf{G}(x, y, 0), \mathbf{G}_{1}(x, y)=\mathbf{G}\left(x, y, m L_{t}\right)$,

$\mathbf{G}_{2}(x, y)=\mathbf{G}\left(x, y, L_{t}\right) 0<m<1,0<x<a, 0<y<b$

the expressions for the constants $\mathbf{A}, \mathbf{B}$ and $\mathbf{C}$ are derived in terms of $\mathbf{P}_{\mathbf{i}}$ and $\mathbf{G}_{\mathbf{i}}(\mathrm{i}=1$

to 3). The general solution of Eqn. (14) is given by:

$q_{n}(\tau)=E_{1 n} q_{n}^{0}+E_{2 n} \dot{q}_{n}^{0}+F_{1 n} a_{n}+F_{2 n} b_{n}+F_{3 n} c_{n}$

where $E_{1 n}, E_{2 n}, \quad F_{1 n}, \quad F_{2 n}, \quad F_{3 n}$ denote complicated mathematical expressions containing $\omega_{n}, \xi_{n}$ and $\tau$ (see [22] ) and

$a_{n}=\iint \mathbf{U}_{n}^{T} \mathbf{A}_{n} d x d y, b_{n}=\iint \mathbf{U}_{n}^{T} \mathbf{B}_{n} d x d y, c_{n}=\iint \mathbf{U}_{n}^{T} \mathbf{C}_{n} d x d y$

The iteration procedure applied to solve the above equations (11) is identical to the ones for circular plates and beams given in $[22,23]$. This is why it is summarised very briefly here.

We first solve the eigen-value problem for the plate described by the small deflection Mindlin plate theory which gives the frequencies of free vibration, the normal modes of vibration $\mathbf{U}_{\mathbf{n}}$, and the necessary derivatives. Then the initial values $q_{n}^{0}$ and $\dot{q}_{n}^{0}$ are computed according to Eqns. (13).

At each time-step $\left[t_{i}, t_{i+1}\right]$ an iteration procedure is applied. It includes solving the small deflection plate theory equations and then using the obtained values of $w$ and its derivatives to compute $G_{u}$ and $G_{v}$, solve the system of algebraic equations (A1,A2) for $u$ and $v$, and then form the nonlinear force vector $\mathbf{G}_{\mathbf{L}}$, and solve the system of ODE (11) again. At the next time step the values of generalized displacement and velocity vectors 
$\mathbf{U}$ and $\dot{\mathbf{U}}$ at the end of the previous time interval are used as initial conditions. The iterative procedure is repeated until the convergence criterion is fulfilled.

The integrals in Eqns. (12, 13 and 17) are evaluated by numerical quadrature following the Simpson rule.

\section{Damage identification technique}

In a lot of cases small damage in structures doesn't influence the static response or the small amplitude vibration response of the structure. However, its influence can be observed when the structure is subjected to large dynamic loads, leading to large amplitude vibrations. In this case even small changes in the structure (like cracks and other local damage scenarios) can have a considerable effect on the structural response in the time domain, which in turn can give an indication of the presence of damage. Damage which induces very small changes in the natural frequencies and the mode shapes may result in phase shifts between the vibration response of the healthy structure and the damaged structure in the time domain. In many applications vibrating plates are subjected to dynamic loadings leading to large amplitude vibrations. In such cases the small deflection plate theory cannot provide adequate simulation of the plate vibration response and therefore the large deflection plate theory should be applied, which takes into account the geometrical nonlinearities present in the system. On the other hand, large amplitude vibrations can increase substantially the influence of small defects (which have very small or nearly no influence on the plate response in the case of small deflections) on the dynamic behaviour of the plate and thus make them easily identifiable. 
There are a lot of techniques to treat nonlinear structural response in the time domain. As mentioned above, the state-space representation of the structural vibration response is a suitable and powerful tool for studying the dynamic behaviour of a structure. A standard technique for dealing with phase space $(w, \dot{w}, t)$ of periodically driven oscillators is to study the projection of $(w, \dot{w})$ at moments in time $t$, where $t$ is a multiple of the period $T=2 \pi / \omega$. Here $\omega$ can be the frequency of the excitation of the mechanical system, an eigen frequency of the structure, or its multiple, and $T$ is a period of the forcing function, an eigen period of the system, or its multiple. The result of inspecting the phase projection $(w, \dot{w})$ only at specific times $t=k T$ is a sequence of dots, representing the socalled Poincaré map. The steady-state converging trajectories, which represent the attractor, are usually formed in the phase space and in many cases of nonlinear systems they are very sensitive to any changes in the system.

The idea of the approach presented here is based on the following considerations:

1. A Poincaré map can be interpreted as a discrete representation of the dynamic system in a state space which is one dimension smaller than the original continuous space of the dynamic system. Since it preserves many properties of periodic and quasiperiodic orbits of the original system and has a lower dimension, it is often used for analyzing the original system.

2. The Poincaré maps contain data for the displacements and the velocities of the structure in a compact form and since these two parameters are expected to be sensitive to damage these diagrams can be used to detect damage. When the damage is large and the plate undergoes substantial nonlinear vibrations, this leads to changes in the attractor of the vibrating system in the phase space and then the application for damage assessment 
purposes becomes obvious. Even when the damage is small, and the responses of the damaged and the healthy structure are close to each other, the points from the Poincaré map are easier to use for comparison and identification purposes because the number of these points is much smaller than the enormous number of points in the time-history diagrams.

According to the above considerations the following damage index can be introduced for the $\mathrm{i}^{\text {-th }}$ node:

$$
\begin{aligned}
& I_{i}^{d}=\frac{S_{i}^{u}-S_{i}^{d}}{S_{i}^{u}}, \\
& S_{i}^{u}=\sum_{j=1}^{N_{p}-1} \sqrt{\left(w_{i, j+1}^{u}-w_{i, j}^{u}\right)^{2}+\left(\dot{w}_{i, j+1}^{u}-\dot{w}_{i, j}^{u}\right)^{2}} \\
& S_{i}^{d}=\sum_{j=1}^{N_{p}-1} \sqrt{\left(w_{i, j+1}^{d}-w_{i, j}^{d}\right)^{2}+\left(\dot{w}_{i, j+1}^{d}-\dot{w}_{i, j}^{d}\right)^{2}}
\end{aligned}
$$

where, $i=1,2 \ldots N_{\text {nodes }}, N_{\text {node }}$ is the number of nodes, $N_{p}$ is the number of points in the Poincaré map and $\left(w_{i j}^{u}, \dot{w}_{i j}^{u}\right)$ and $\left(w_{i j}^{d}, \dot{w}_{i j}^{d}\right)$ denotes the $\mathrm{j}^{\text {th }}$ point on the Poincaré maps of the undamaged and the damaged states, respectively.

A small (close to 0) damage index will indicate no damage, while a big damage index will indicate the presence of a fault at the corresponding location. The above damage index depends on the location of the point on the plate, and consequently it is a function of the plate coordinates $x$ and $y$. One can expect that the maxima of the surface $I^{d}\left(x_{d}, y_{d}\right)(18 \mathrm{a})$ will represent the location of the damage, i.e. $I_{\max }^{d}\left(x_{d}, y_{d}\right)=\max _{i}\left\{I_{i}^{d}\right\}$

It is easy to notice that $S_{i}^{u}$ and $S_{i}^{d}(18 \mathrm{~b}, \mathrm{c})$ represent the lengths of the lines formed by connecting the dots on the Poincaré maps for the damaged and the non-damaged plate 


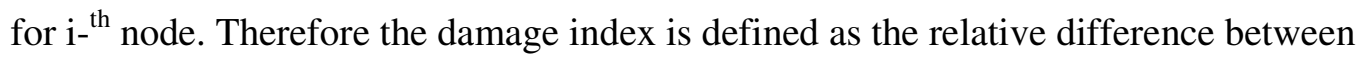
these two lengths. The logical expectations are that:

1) Since the fault influences the vibration response of the plate it will introduce changes in the Poincaré map. The differences between the Poincaré maps of the healthy and damaged plates will be indicative of the presence of damage.

2) At the nodes close to the damaged area the introduced damage index $I_{i}^{d}$ (18 a) will be larger than the index for points which are far from the damaged zone. This can be used to localize the detected damage.

\section{THE CASE STUDY}

This study focuses on damage assessment in rectangular and square plates. The material characteristics of the plates are: Young modulus $E=7.10^{10} \mathrm{~N} / \mathrm{m}^{2}$, Poison ratio $v=0.34$, density $\rho=2778 \mathrm{~kg} / \mathrm{m}^{3}$. The damping coefficient $c_{1}=c_{2} \frac{12}{h^{2}}$ in Eqns ( 7 ) was chosen to be $0.00075 \frac{N s}{m^{3}}$. The square plate has dimensions $a=b=0.5 \mathrm{~m}$ and thickness $h=0.006 \mathrm{~m}$. Two cases of damage were considered: - A) almost central damage- thickness reduction in a small area located in the central part of the plate slightly moved from the centre (one element up and one element left) (see Fig. 2); B) side damage- thickness reduction in a small area close to the left lower corner of the plate as shown in Fig. 2. A finite element model of the plate is shown in Fig. 2.

The finite element discretization of the rectangular plate is shown in Fig. 3. Its dimensions are : $a=10 \mathrm{~m}, b=2.5 \mathrm{~m}, h=0.05 \mathrm{~m}$. Again two damage cases are considered: A) almost central damage and B) side damage. The damage is modelled as thickness 
reduction. In both cases (rectangular and square plates) the thicknesses of the damaged areas are equal to half the thickness of the undamaged plate.

The aim of the following numerical example performed is to test the suggested procedures to detect and localise damage in the plate.

\section{SOME RESULTS}

In this paragraph some result for the detection and the localisation of the defects described above in the square and the rectangular plates considered are discussed.

First of all the sensitivity of the first ten natural frequencies of the plate was tested. Our results show that in these particular cases both defects introduce very small or nearly no changes in the first 10 natural frequencies of the plate. The relative differences between the frequencies of the intact and the damaged square and rectangular plates are shown in Figs. 4 and 5, respectively. Especially for the case B) the differences between the frequencies are very small and are in the range of the accuracy of its experimental determination. So in these particular cases there is obviously a need for an alternative method.

Then, the forced response of the plates subjected to harmonic loading was considered. Cases when the frequency of excitation is close to one of the first natural frequencies of the system are interesting and important for this study of a nonlinear system because they often lead to complex phenomena like beating, quasi-periodic or chaotic vibrations [24]. In such regimes the vibrating systems are usually quite sensitive to even small changes introduced in their geometry and/or the physical properties including damage. These regimes of large amplitude vibrations are therefore expected to enhance the sensitivity of nonlinear vibrating structures to damage, even though the changes in the natural frequencies might be negligible. Accordingly the plates considered were 
subjected to a harmonic loading uniformly distributed over the plate surface. Numerical experiments were carried out for different values of the excitation frequency.

In the case of a square plate with a central defect (case A) the excitation frequency was chosen equal to $\omega_{e}=1000 \mathrm{rad} / \mathrm{s}$. In order to show the applicability of the methods for higher frequencies for the case $\mathbf{B}$ the excitation frequency was $\omega_{e}=2000 \mathrm{rad} / \mathrm{s}$. (The first two natural frequencies of healthy plate are $\omega_{1}=1326.32 \mathrm{rad} / \mathrm{s}, \omega_{2}=2700.3 \mathrm{rad} / \mathrm{s}$ ) The amplitude of the harmonic loading was $6 \mathrm{~N}$.

Let us first have a look at the time histories of the plate response for the undamaged and the damaged cases. Figures 6 and 7 give parts of the time histories for the case of central defect and side defect compared to those for the non damaged plate. It can be observed that the applied load leads to large amplitude vibrations of the plate. Due to the fact that the excitation frequency is close to the first natural frequency of the plate a beating phenomenon occurs. It can be appreciated from Figures 6 and 7 that the time histories undergo changes with damage. As expected, the differences for case A central defect are bigger than the differences for case $\mathbf{B}$ side defect. It can be seen that close to the origin $(t=0)$ the responses almost coincide with each other (especially for case B) but then the phase shifts and the differences between the responses increase.

The next question is how to use these differences in the time responses of the nondamaged and the damaged plate for the purposes of VSHM. Our approach suggests the use of Poincaré maps and the damage index $I_{i}^{d}$ (equation 18 a) which is based on these maps to detect and localise the damage. To visualize the damage index and to set a threshold for detecting the damage we use the so-called contour plots. A contour plot is a graphical technique for representing a 3-dimensional surface by plotting constant $z$ 
slices, called contours, on a 2-dimensional plane. That is, for a given value of $z$, lines are drawn that connect the $(x, y)$ coordinates that correspond to this particular value of $z$.

The influence of the damage on the Poincare maps at the plate centre can be seen in Figures 8 and 9. As can be expected the influence of the central damage (case A) on the Poincaré maps of the plate is larger than the influence of the side defect (case B). The introduced damages do not change the type of the Poincare section (circle) they only influence the length of the curves formed by the Poincaré dots. Then the damage index $I_{i}^{d}$ was calculated for the points from the Poincaré maps for all the nodes and its contour plots were obtained. Fig. 10 details the contour plots of $I_{i}^{d}$ for case $\mathbf{A}$ defect for all the plate nodes. As can be seen from this plot case A damage can be detected and localised quite precisely.

Fig. 11 presents analogous contour plots for the case of side damage (case B). It can be observed that the plot identifies quite precisely the position of the defect in spite of the fact that the absolute values of the differences in the displacements and the velocities of the two responses at the nodes of the damaged area are small. The calculations for smaller values of damage $\left(h_{\text {damaged }} / h=0.66\right)$ still show very good prediction of the damage location (not shown here).

Analogous results were obtained for the case of the rectangular plate.

The time history diagram of the plate centre of a plate with a side defect is shown in Fig. 12. The excitation frequency is $260 \mathrm{rad} / \mathrm{s}$, which is only $7 \%$ less then the first eigen frequency of the healthy plate. Again a strong beating can be observed in the responses of the healthy and damaged plates. The phase of the response of the damaged plate and 
the difference between the responses increases with the time. In spite of the fact that the Poincaré maps at the plate centre (where the deflections and the velocity are the largest) are very close to each others (Fig. 13) the damage index corresponding to the damaged area has the biggest values, which gives the possibility to locate the damage. In Fig. 14 the contour plot of $I_{i}^{d}$ is compared to the FE model of the plate where the damaged area is coloured in white. It can be seen that the damage location is predicted very precisely. The same conclusion applies in the case of the rectangular plate with central damage (see Figs. 15 and 16). As in the case of the square plate the location of the damage is predicted very precisely and even the small non-symmetry of the damage location with respect to the plate centre can be seen on the contour map of $I_{i}^{d}$.

\section{CONCLUSIONS}

A numerical approach for studying the geometrically nonlinear vibrations of rectangular plates with and without damage is developed in the paper. The computed time domain responses are used to analyse the dynamic behaviour of plates in the intact condition and of plates with defects. Based on these analyses a damage index and a method for damage detection and damage location have been proposed. The damage assessment method is based on the phase space representation of the time domain nonlinear response of the plate and uses the analysis of the Poincare map of the response. The developed damage assessment procedure was applied for the test cases of a square and a rectangular aluminium plates with different defects modelled as areas with reduced thickness. It was demonstrated that damage can influence substantially the time domain response of the plate despite its very small influence on the plate natural frequencies. The suggested damage assessment method demonstrates quite good capability for detecting damage. The index suggested in Eqns. (18) is used to localise damage and it shows very good 
performance. The potential, the sensitivity and the applicability of the developed method still have to be tested for real measurements and for more structures, defects and loading conditions.

\section{ACKNOWLEDGMENTS}

The authors would like to acknowledge the support of grant 2006/R2-IJP from the Royal Society, UK and of the Bulgarian Research Fund for the partial support through grant TN-1518/2005. 


\section{APPENDIX. Finite difference scheme for solution of equations for mid-plane}

\section{displacements.}

Applying the central difference formula the equations ( $7 \mathrm{a}, \mathrm{b})$ are transformed into the following system of algebraic equation with respect to $u_{i, j}, v_{i, j}, i=1,2, \ldots N_{x}, j=1,2, \ldots N_{y}$, ( $N_{x}$ and $N_{y}$ are the numbers of nodes along the axis x and y , correspondingly.

$$
\begin{aligned}
& A_{i+\frac{1}{2}, j} u_{i+1, j}+A_{i-\frac{1}{2}, j} u_{i-1, j}-\left(\frac{A_{i+\frac{1}{2}, j}+A_{i-\frac{1}{2}, j}}{\Delta_{x}^{2}}+\frac{1-v}{2} \frac{A_{i, j+\frac{1}{2}}+A_{i, j-\frac{1}{2}}}{\Delta_{y}^{2}}\right) u_{i, j}+\frac{1-v}{2} \frac{A_{i, j+\frac{1}{2}}}{\Delta_{y}^{2}} u_{i, j+1}+ \\
& \frac{1-v}{2} \frac{A_{i, j-\frac{1}{2}}}{\Delta_{y}^{2}} u_{i, j-1}+\frac{v A_{i+\frac{1}{2}, j}+\frac{1-v}{2} A_{i, j+\frac{1}{2}}}{4 \Delta_{x} \Delta_{y}} v_{i+1, j+1}-\left(\frac{v A_{i+\frac{1}{2}, j}+\frac{1-v}{2} A_{i, j-\frac{1}{2}}}{4 \Delta_{x} \Delta_{y}}\right) v_{i-1, j+1}- \\
& \left(\frac{v A_{i-\frac{1}{2}, j}+\frac{1-v}{2} A_{i, j+\frac{1}{2}}}{4 \Delta_{x} \Delta_{y}}\right) v_{i+1, j-1}+\left(\frac{v A_{i-\frac{1}{2}, j}+\frac{1-v}{2} A_{i, j+\frac{1}{2}}}{4 \Delta_{x} \Delta_{y}}\right) v_{i-1, j-1}=G_{i, j}^{u} v_{i, j}
\end{aligned}
$$

$$
\left(\frac{v A_{i, j+\frac{1}{2}}+\frac{1-v}{2} A_{i+\frac{1}{2}, j}}{4 \Delta_{x} \Delta_{y}}\right) u_{i+1, j+1}-\left(\frac{v A_{i, j+\frac{1}{2}}+\frac{1-v}{2} A_{i+\frac{1}{2}, j}}{4 \Delta_{x} \Delta_{y}}\right) u_{i-1, j+1}+\left(\frac{v A_{i, j-\frac{1}{2}}+\frac{1-v}{2} A_{i-\frac{1}{2}, j}}{4 \Delta_{x} \Delta_{y}}\right) u_{i-1, j-1}
$$

$$
-\left(\frac{v A_{i, j+\frac{1}{2}}+\frac{1-v}{2} A_{i-\frac{1}{2}, j}}{4 \Delta_{x} \Delta_{y}}\right) u_{i+1, j-1}+\frac{A_{i, j+\frac{1}{2}}}{\Delta_{y}^{2}} v_{i, j+1}-\left(\frac{A_{i, j+\frac{1}{2}}+A_{i, j-\frac{1}{2}}}{\Delta_{y}^{2}}+\frac{\frac{1-v}{2} A_{i+\frac{1}{2}, j}+\frac{1-v}{2} A_{i-\frac{1}{2}, j}}{\Delta_{x}^{2}}\right) v_{i, j}
$$$$
+\frac{A_{i, j-\frac{1}{2}}}{\Delta_{y}^{2}} v_{i, j-1}+\frac{1-v}{2} \frac{A_{i+\frac{1}{2}, j}}{\Delta_{x}^{2}} v_{i+1, j}+\frac{1-v}{2} \frac{A_{i-\frac{1}{2}, j}}{\Delta_{x}^{2}} v_{i-1, j}=G_{i, j}^{v}
$$

Assuming $G_{u}$ and $G_{v}$ are known functions the linear system of algebraic equations is solved by using LSLRG routine of the Microsoft IMSL Libraries. 


\section{REFERENCES}

[1] P.F. Rizos, N. Apragathos, Identification of crack location and magnitude in a cantilevered beam from the vibration modes. Journal of Sound and Vibration 138 (1990), 381-388.

[2] C. Dong, P. Q. Zhang, W. Q. Feng, T. C. Huang, The sensitivity study of the modal parameters of a cracked beam, Proceeding of the $12^{\text {th }} I M A C$, Honolulu, HW (1994), pp. 98-105.

[3] H.T. Banks, D.J. Inman, D.J. Leo, Y. Wang, An experimentally validated damage detection theory in smart structures. Journal of Sound and Vibration 191 (1996), 859-880.

[4] J. Chance, G.R. Tomlinson, K. Worden, A simplified approach to the numerical and experimental modelling of the dynamics of a cracked beam, Proceedings of the $12^{\text {th }}$ IMAC, Honolulu, HW (1994), pp. $778-786$.

[5] Y. Yam, D. S. Bayard, R. E. Scheid, Frequency domain identification for robust large space structures control design, ACC Proceedings, San Francisco, C A (1993), pp. 3021-3023.

[6] P. Verboven, E. Parloo, P. Guillaume, M. Van Overmeire, Autonomous structural health monitoring - part I: modal parameter estimation and tracking. Mechanical Systems and Signal Processing 16 (2002), 637-657.

[7] S.W. Doebling, C.R. Farrar, M.B. Prime, D.W. Shevitz, Damage Identification and Health Monitoring of Structural Systems from Changes in Their Vibration Characteristics: A Literature Review, (Report LA-12767-MS,Los Alamos National Laboratory, NM, USA (1996).

[8] J. Cattarius, D.J. Inman, Time domain analysis for damage detection in smart structures. Mechanical Systems and Signal Processing 11 (1997), 409-423.

[9] M Todd., J M Nichols, L M Pecora and L Virgin, Vibration-based Damage Assessment Utilizing State Space Geometry Changes: local Attractor Variance Ratio, Smart Mater. Struct. 10 (2001), 1000-1008.

[10] H. T. Banks, D. J. Inman, D.J Leo, Y. Wang, An experimentally validated damage detection theory in smart structures, Journal of Sound and Vibration. 191 (1996), 859-880.

[11] S.L. Tsyfansky, V.I. Beresnevich, Non-linear vibration method for detection of fatigue cracks in aircraft wings. Journal of Sound and Vibration, 236 (2000), 4960 .

[12] U. Andreaus, P. Casini, F. Vestroni, Non-linear dynamics of a cracked beam under harmonic excitation. International Journal of Nonlinear Mechanics, 42 (2007), 566-575.

[13] L Moniz., J.M Nichols., C.J Nichols., M Seaver, S.T. Trickey, M.D. Todd, L.M. Pecora, L.N. Virgin, A multivariate, attractor-based approach to structural health monitoring, Journal of Sound and Vibration 283 (2005), 295-310. 
[14] I. Trendafilova, E. Manoach, M.P. Cartmell, Ostachowicz W., Zak A., An investigation on damage detection in aircrafts panels using nonlinear time series analysis. Key Engineering Materials, 347 (2007), 213-218.

[15] A.C. Okafor, K. Chandrashekhara, Y.P. Jiang, Location of impact in composite plates using waveform-based acoustic emission and gaussian cross-correlation techniques, in: Proceedings of the SPIE, Vol. 2718 (1996), pp. 291-302.

[16] J.B. Bodeux, J.C. Golinval, Modal identification and damage detection using the data-driven stochastic subspace and ARMAV methods. Mechanical Systems and Signal Processing 17 (2003), 83-89.

[17] C.M. Bishop, Novelty detection and neural network validation, in IEE Proceedings: Vision, Image, and Spectral Processing 141 (1994), 217-222.

[18] Z. Hou, M. Noori, R.St. Amand, Wavelet-based approach for structural damage detection. Journal of Engineering Mechanics 126 (7) (2000), 677-683.

[19] J. Grabowska, M. Palacz, M. Krawczuk, W. Ostachowicz, I. Trendafilova, E. Manoach, M. Cartmell, Wavelet analysis for damage identification in composite structures. Key Engineering Materials, Vol. 347 (2007), 253-258.

[20] A. Nag, D.R. Mahapatra, S. Gopalakrishnan, Identification of delamination in composite beams using spectral estimation and a genetic algorithm. Smart Materials and Structures 11 (2003), 899-908.

[21] A.R. Kukreti, H.I. Issa, Dynamic analysis of nonlinear structures by pseudonormal mode superposition method. Computers and Structures, 19 (1984), 653663.

[22] E. Manoach, Dynamic large deflection analysis of elastic-plastic Mindlin circular plates. International Journal of Non-Linear Mechanics, 29, (1994), 723-735.

[23] E.Manoach, Dynamic large deflection analysis of elastic-plastic beams and plates. Proceedings of $7^{\text {th }}$ International Conference Recent Advances on Structural Dynamics, Southampton, July 2000, Vol. 1, pp. 389-400.

[24] P. Ribeiro, E. Manoach, The effect of temperature on the large amplitude vibrations of curved beams. Journal of Sound and Vibration, 285 (2005), 10931107.

[25] J.R Hutchinson, A comparison of Mindlin and Levinson plate theories. Mechanics Research Communications. 14 (1987), 165-170. 


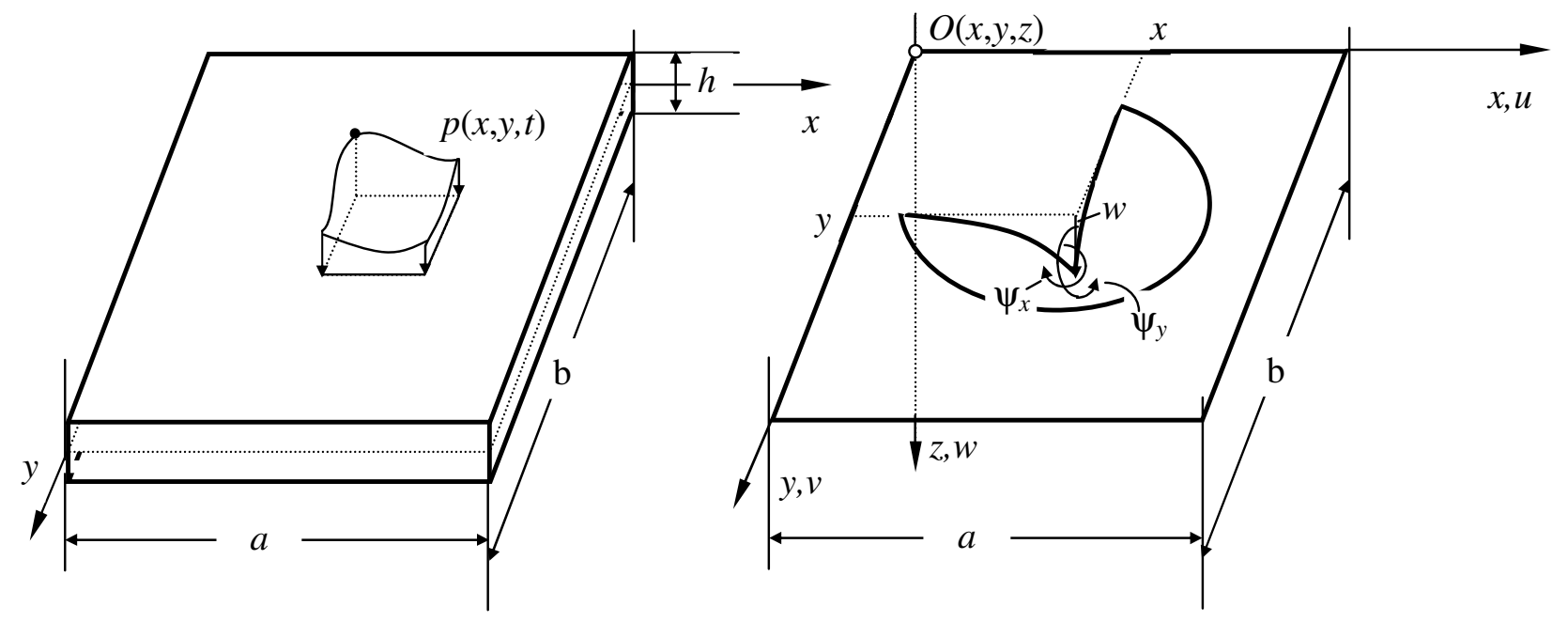

Fig 1 a

Fig. 1 b

Figure 1. 


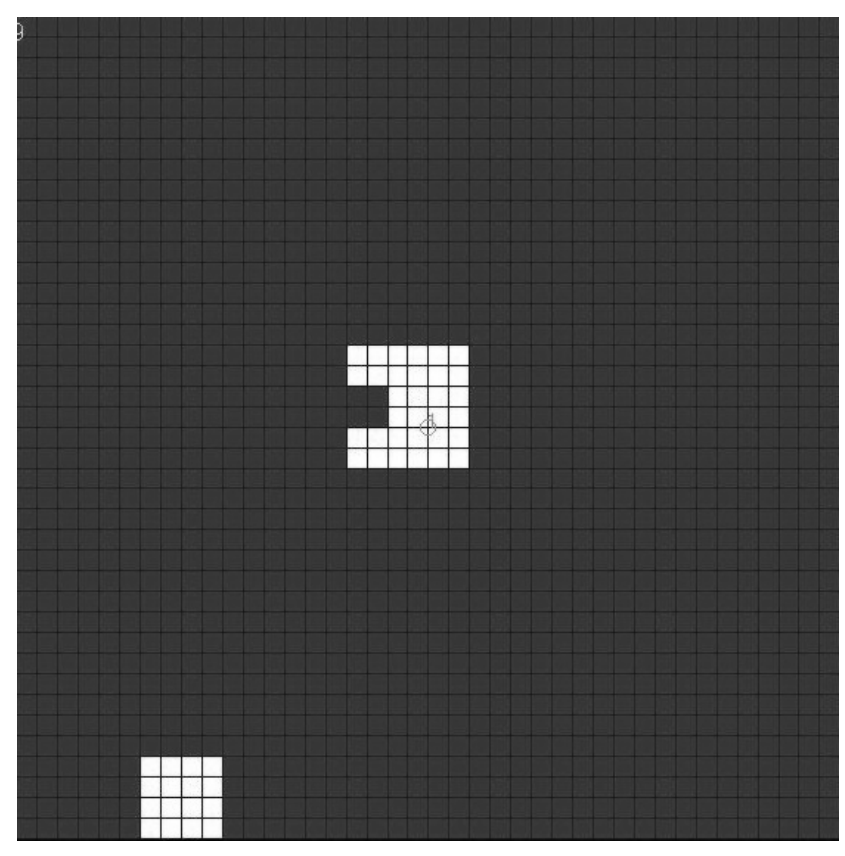

Figure 2 


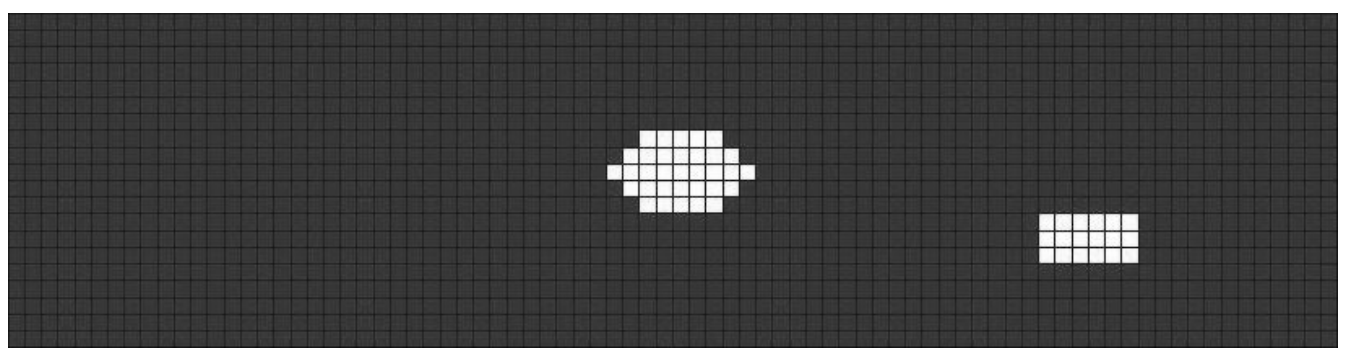

Figure 3. 


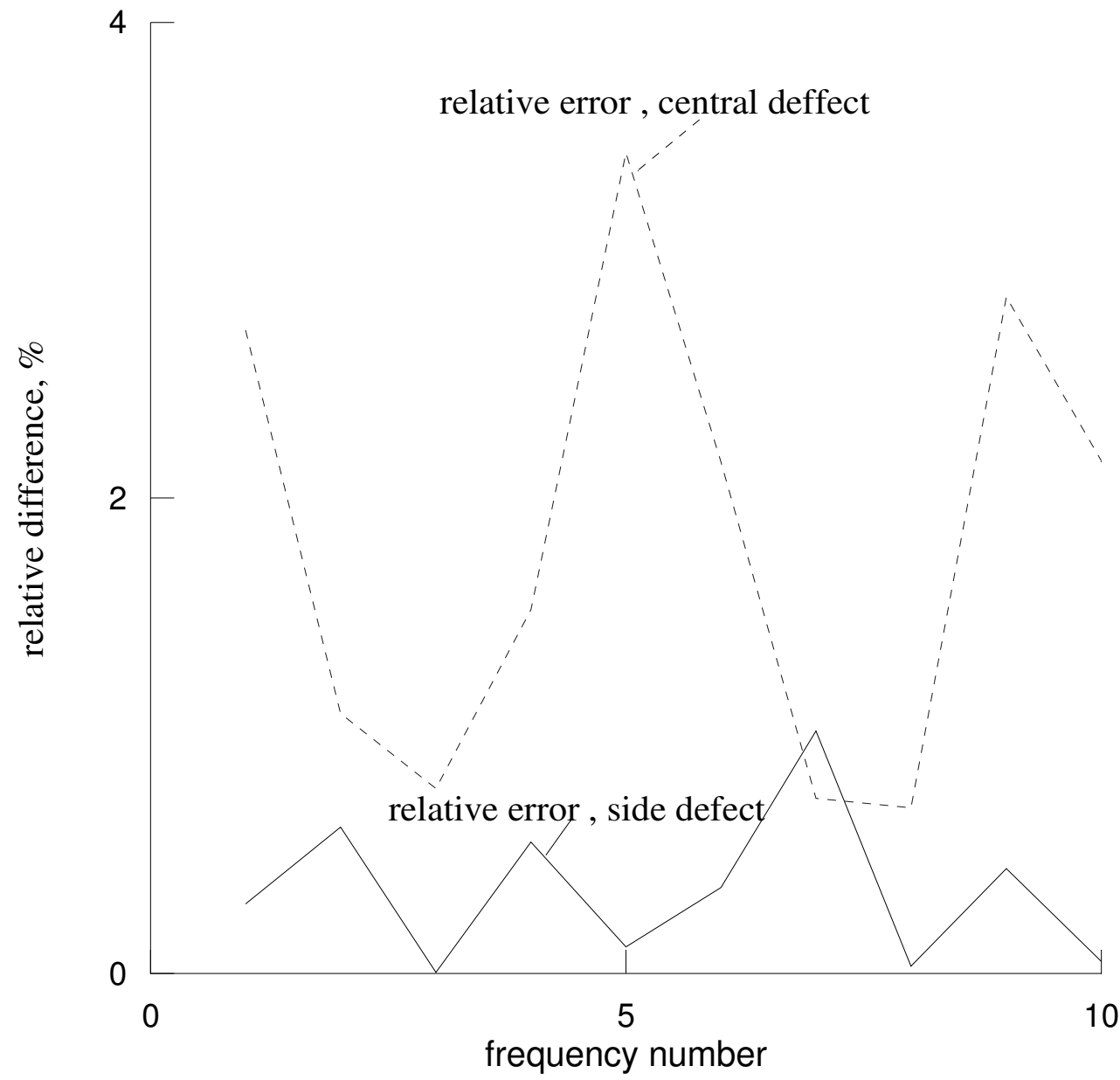

Figure 4. 


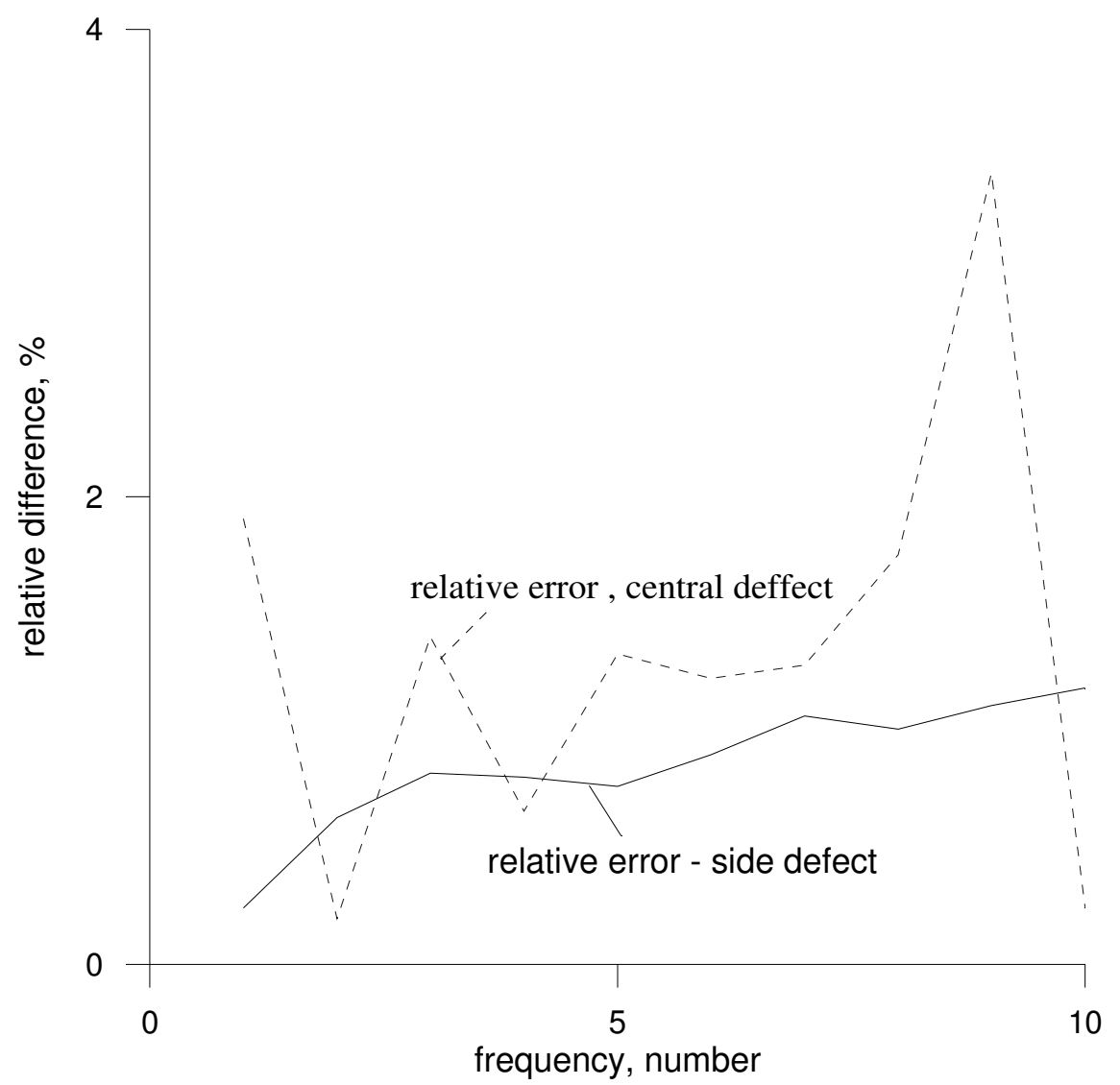

Figure 5. 


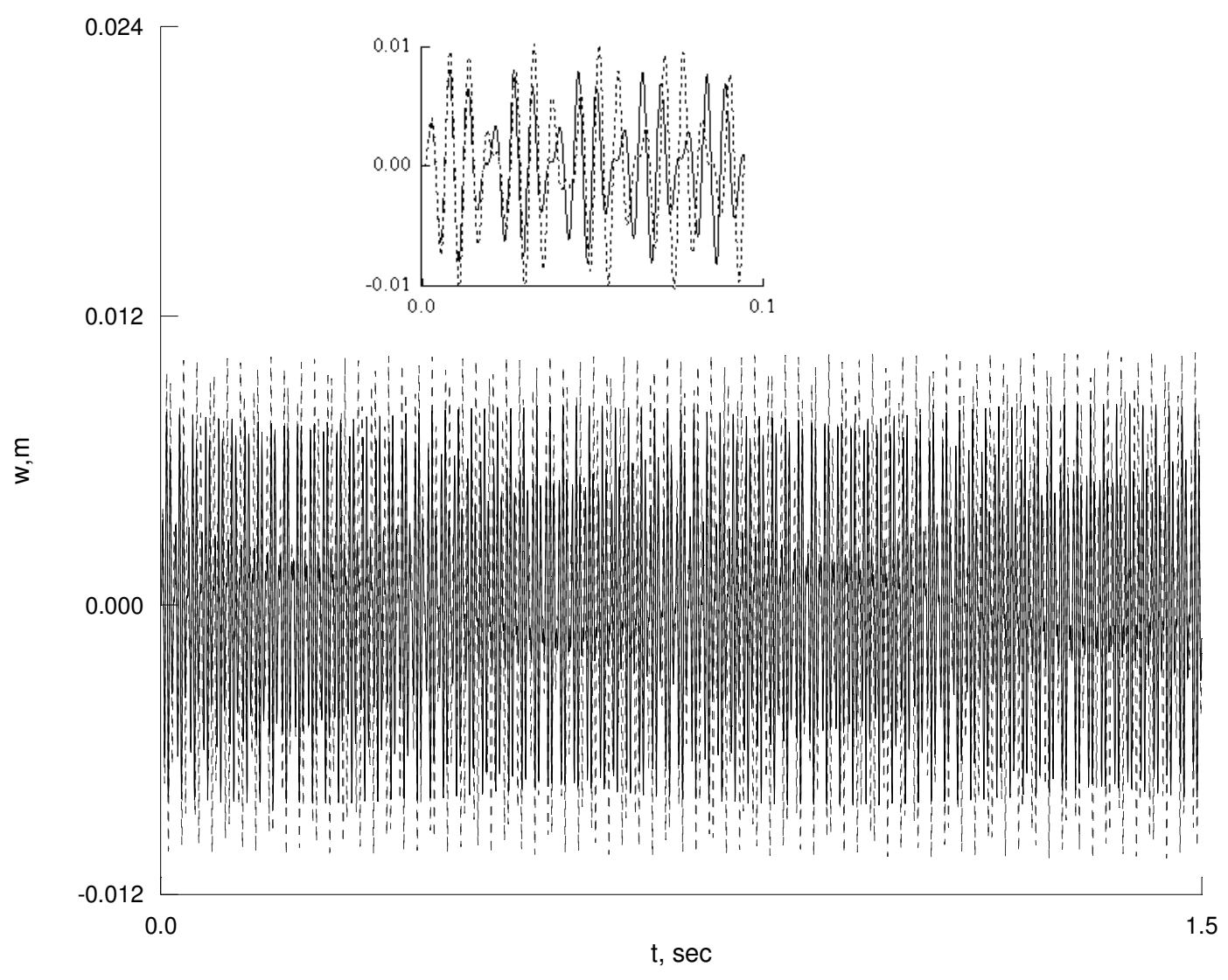

Figure 6. 


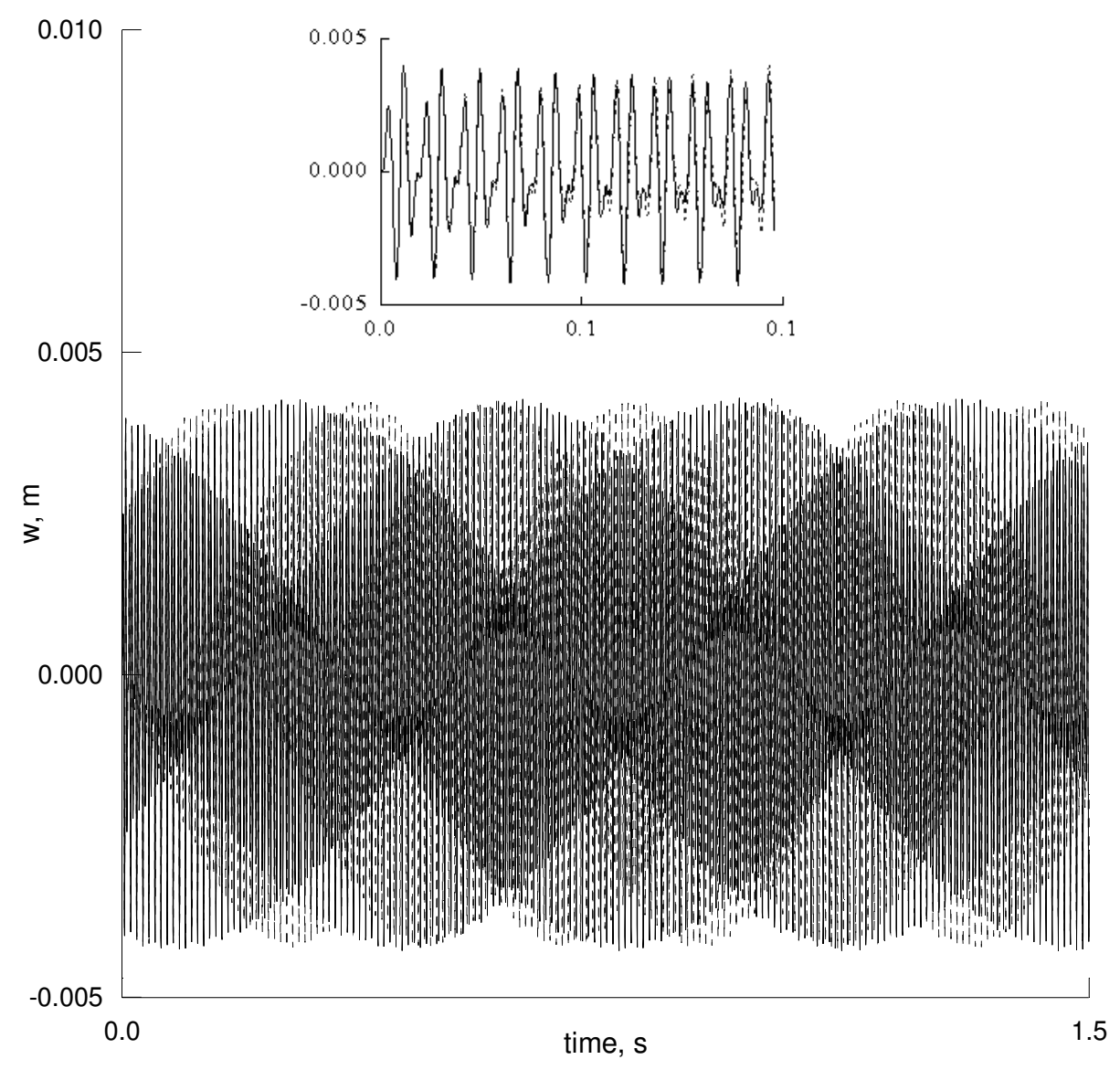

Figure 7. 


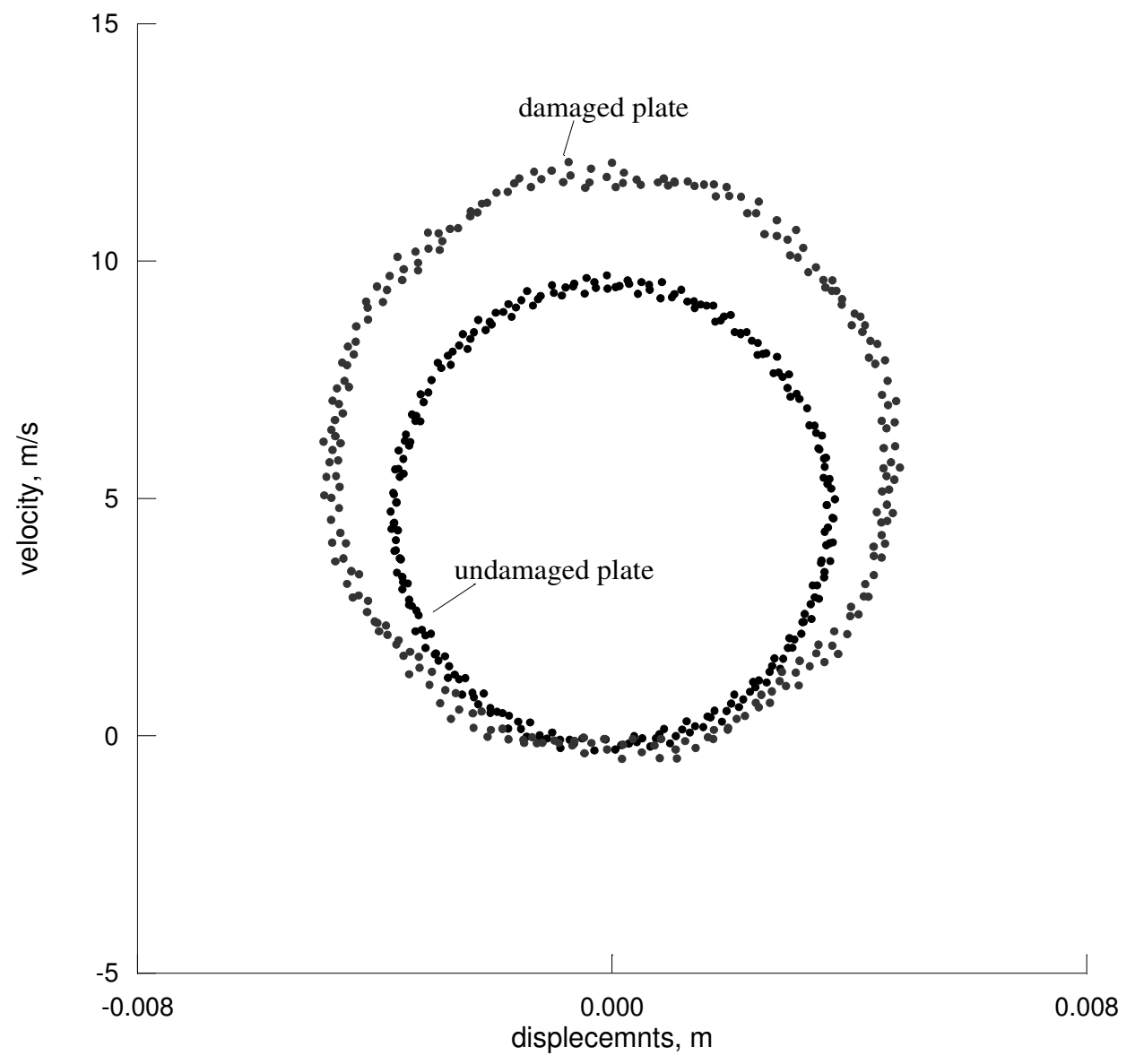

Figure 8. . 


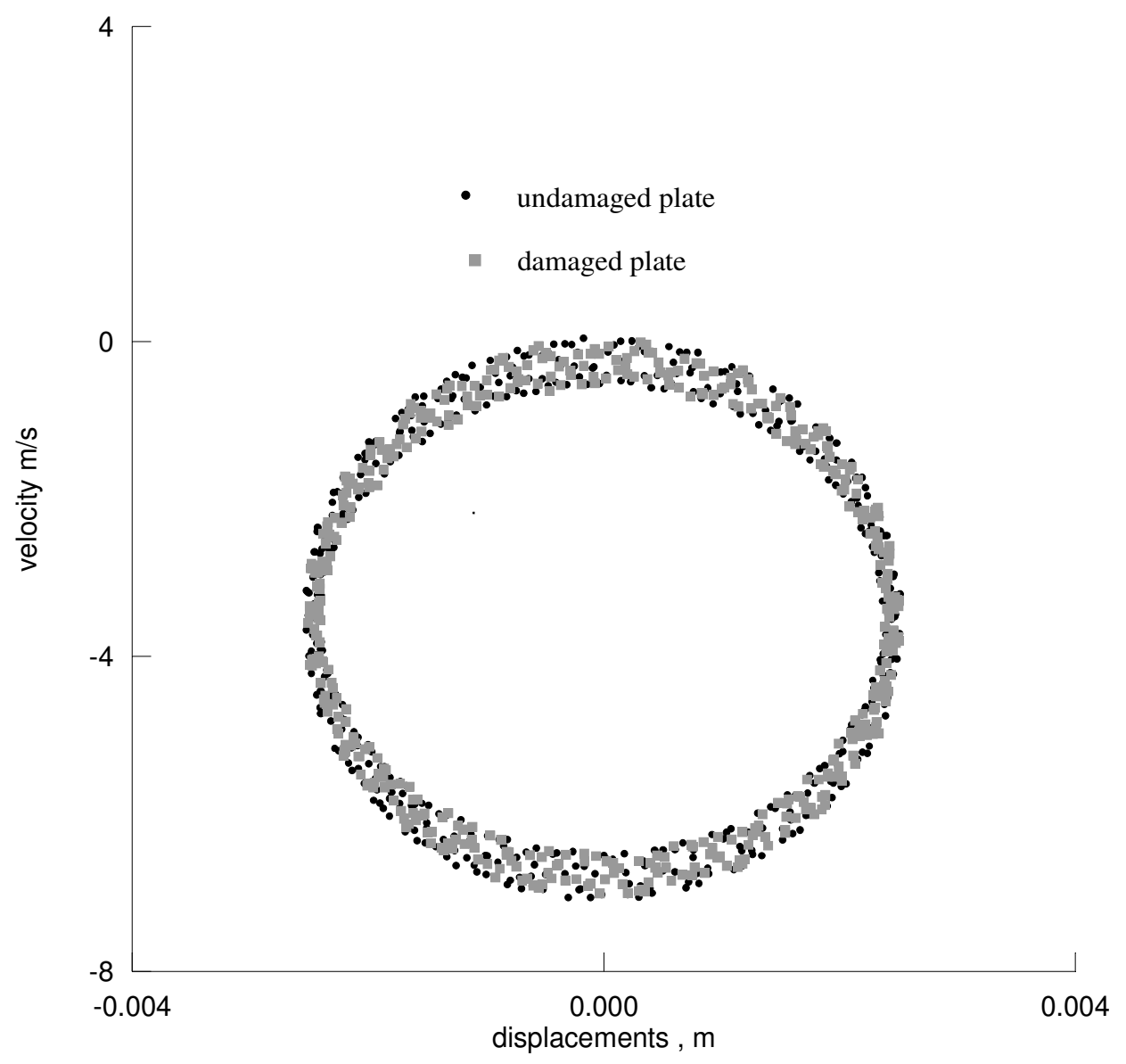

Figure 9. 


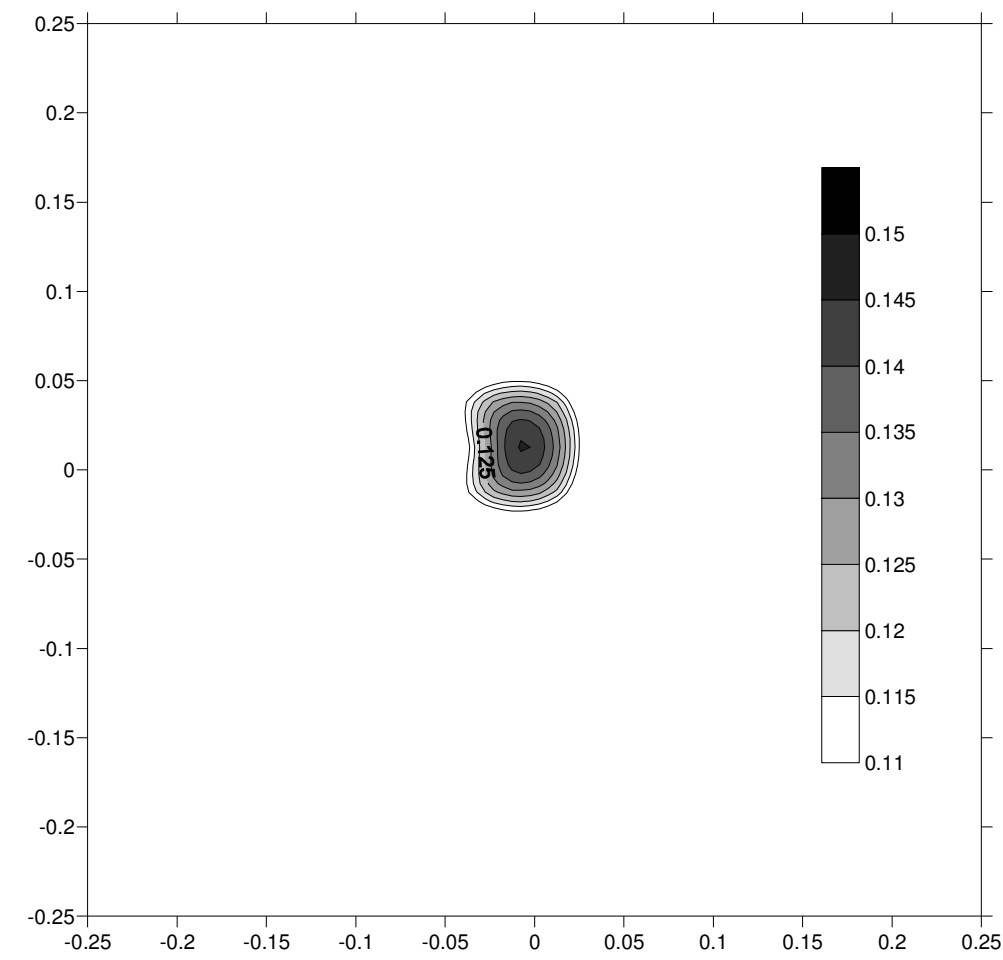

Figure 10. 


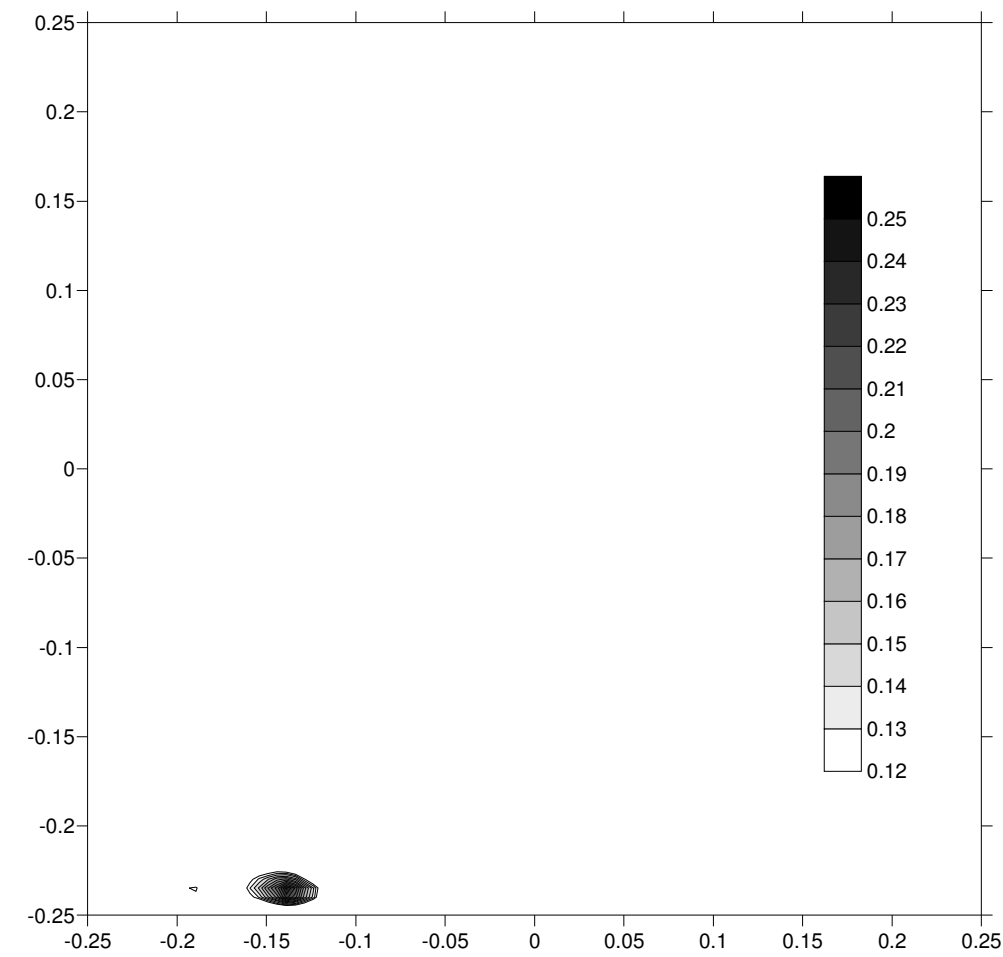

Figure 11 


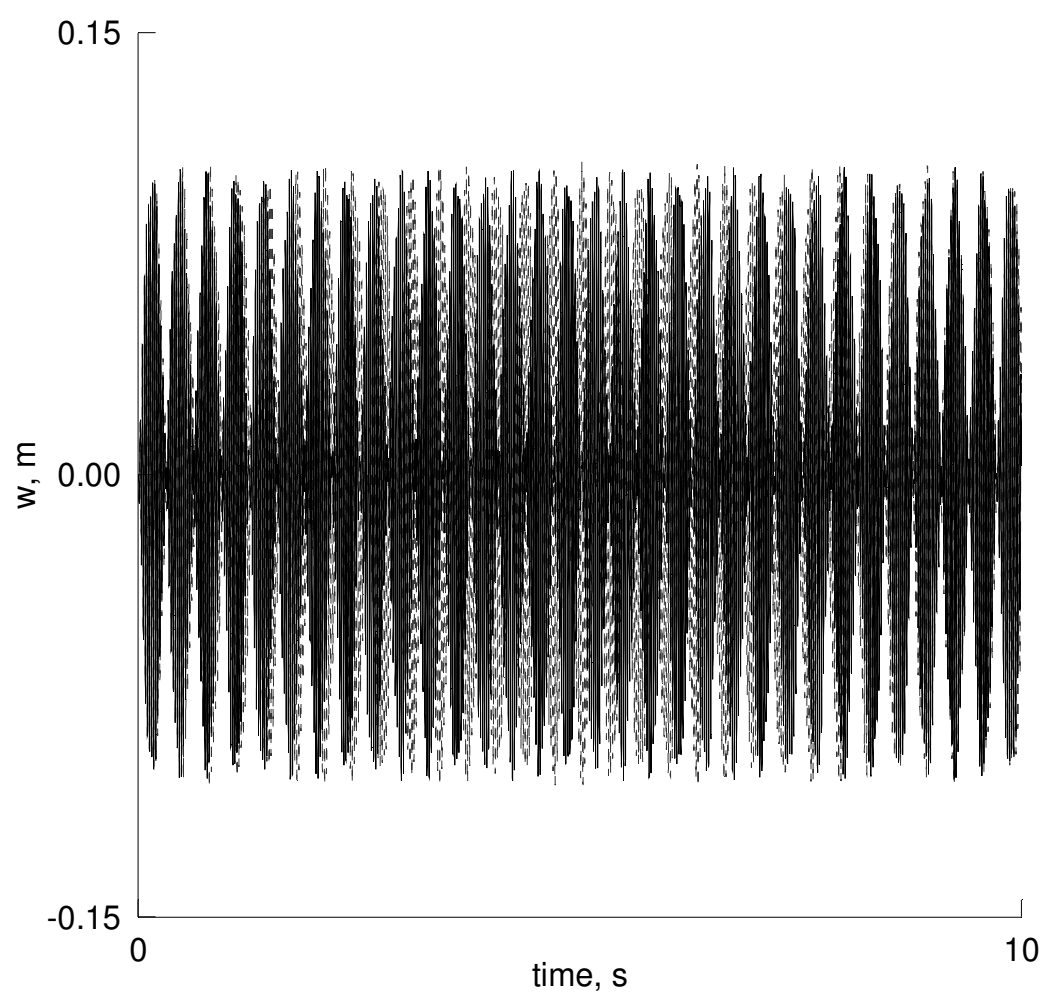

Figure 12. 


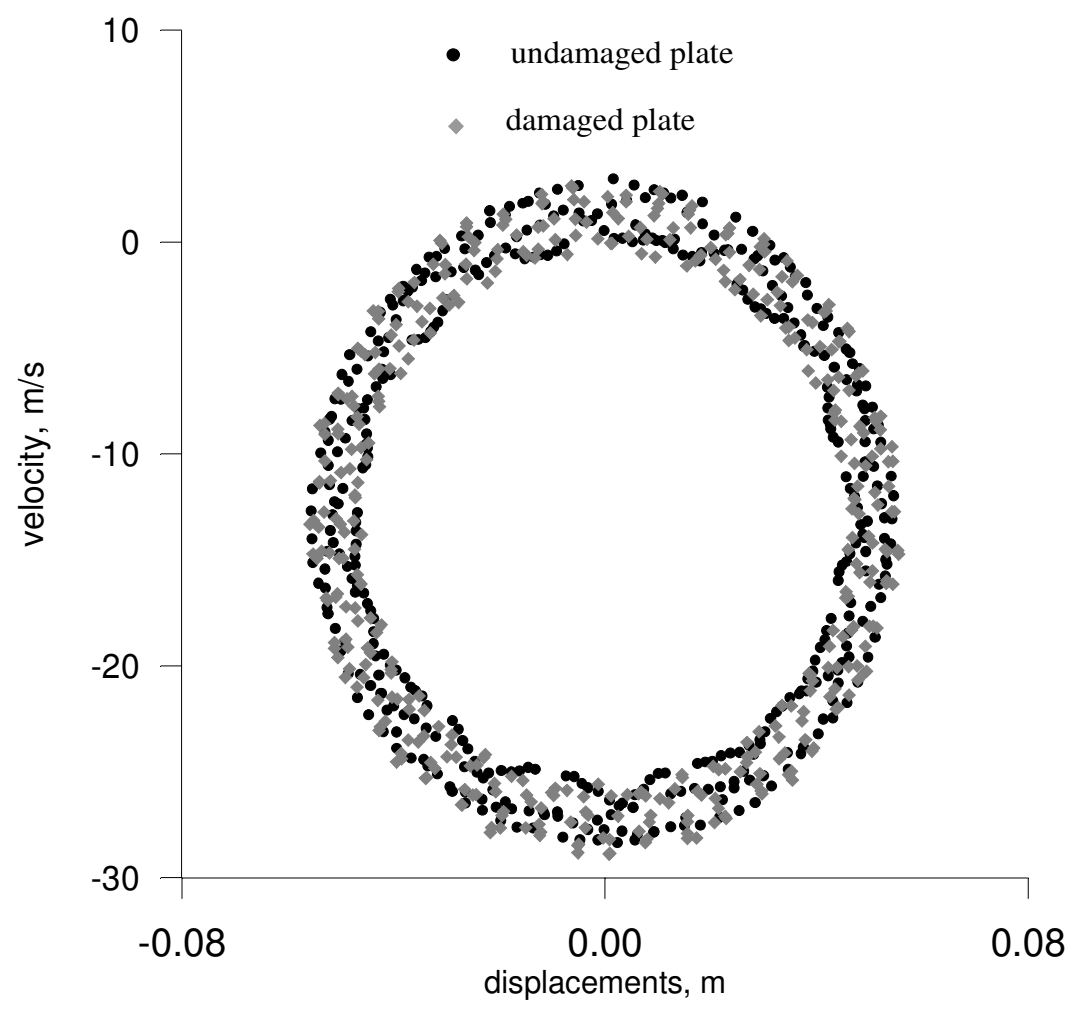

Figure 13. 


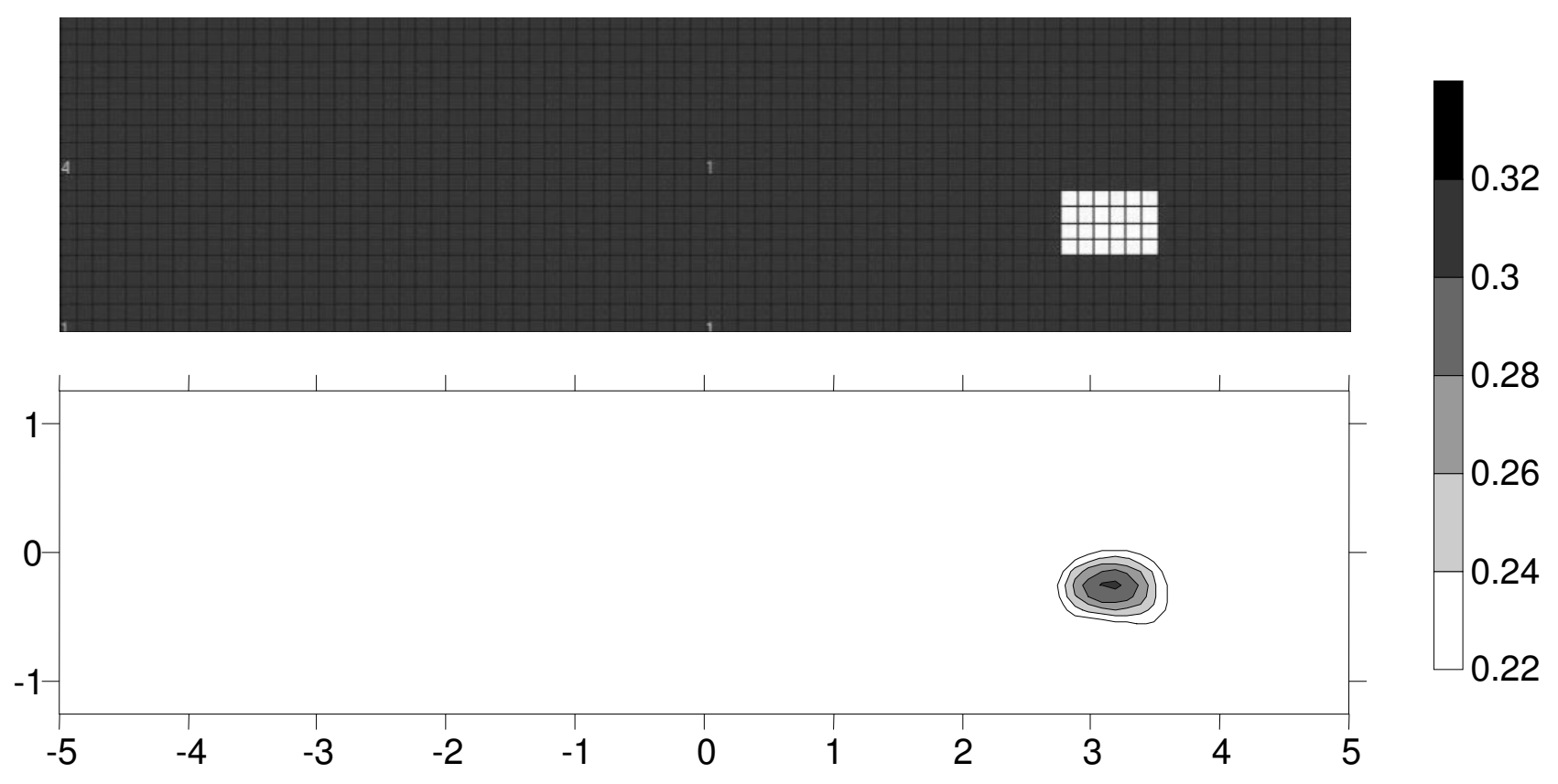

Figure 14. 


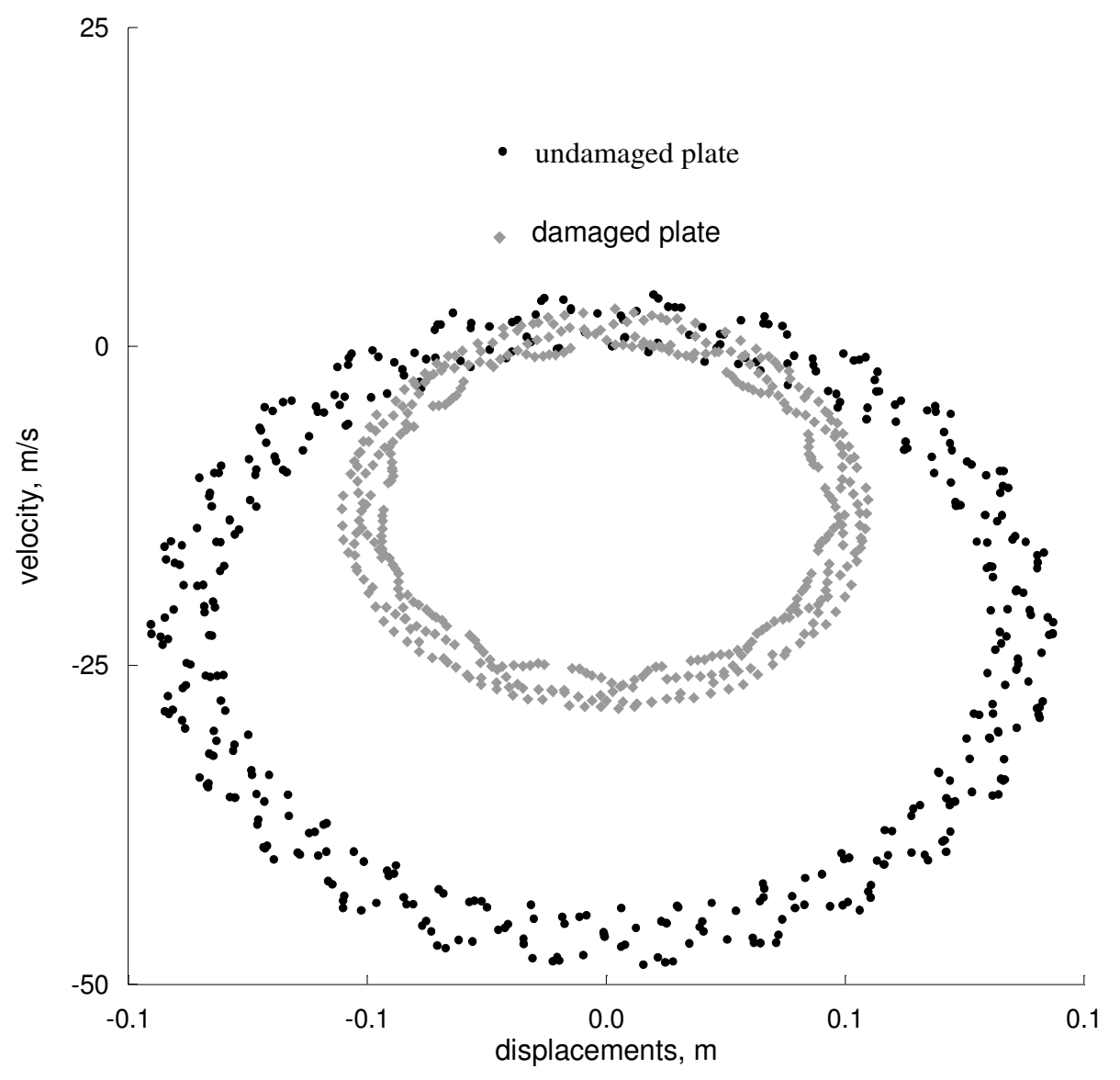

Figure 15. 


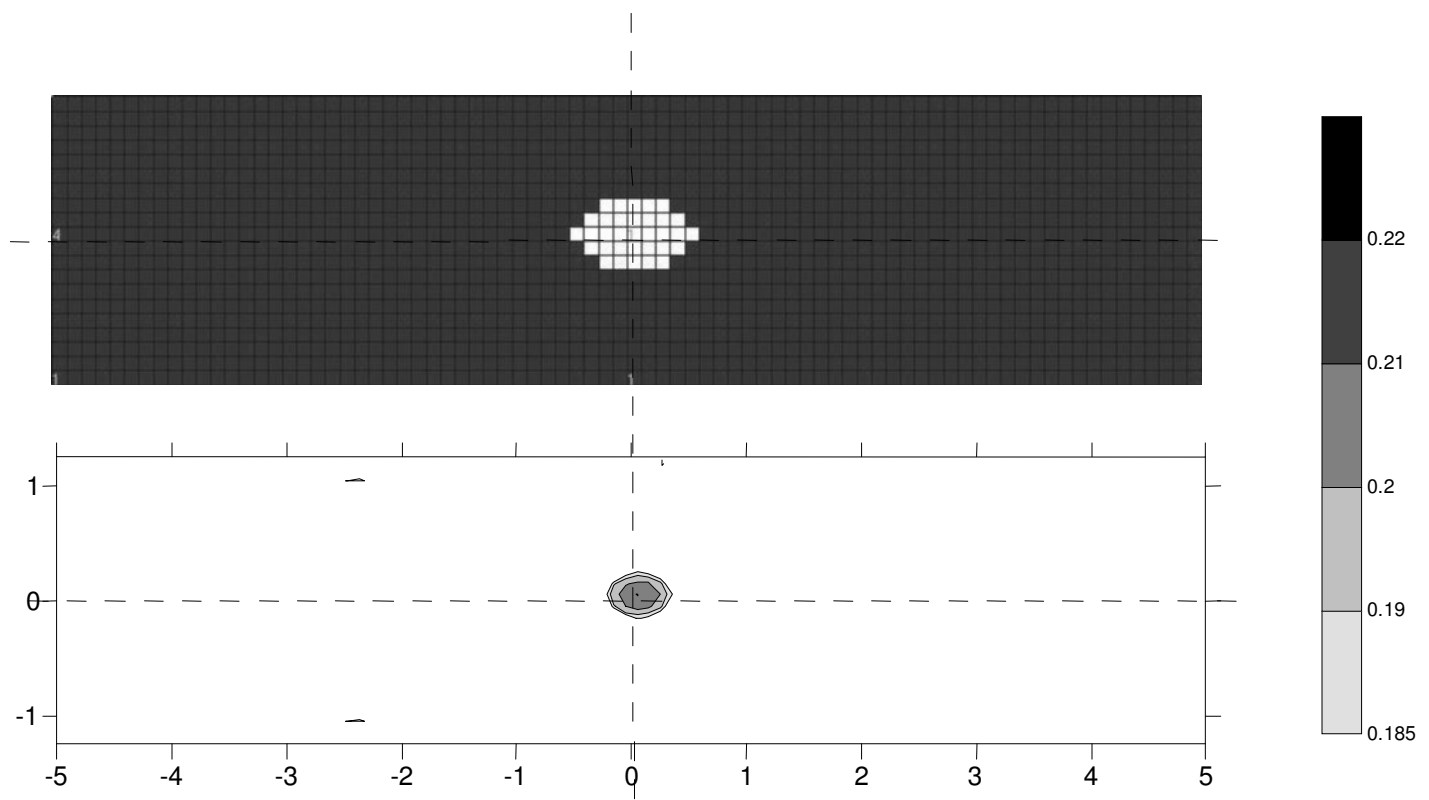

Figure 16. 


\section{FIGURE CAPTIONS}

Figure 1. . Plate geometry and coordinate system. a) Plate dimensions and loading, b) Mid-plane of the plate and the components of the generalized displacement vector.

Figure 2. Square plate with central defect and side defect

Figure 3. Rectangular plate with central defect and side defect

Figure 4. Differences between the eigen frequencies of the damaged and undamaged square plate. Solid line - side defect; dashed line - central defect

Figure 5. Differences between the frequencies of the damaged and undamaged rectangular plate in the case of central and side defects

Figure 6. Time histories of the plate centre in the case of central defect solid line undamaged plate; dashed line - damaged plate. Excitation frequency $\omega_{\mathrm{e}}=1000 \mathrm{rad} / \mathrm{s}$, $\mathrm{p}=6 \mathrm{~N}$.

Figure 7 Time histories of the plate centre of the square plate for the case of side defect. Solid line -undamaged plate; Dashed line - damaged plate. Excitation frequency $\omega_{\mathrm{e}}=2000 \mathrm{rad} / \mathrm{s}, \mathrm{p}=6 \mathrm{~N}$.

Figure 8. Poincaré map of the centre of the plate in the case of central damage.

Figure 9. Poincaré map of the centre of the plate in the case of side damage.

Figure 10. Contour map of damage index $I^{d}$ for central damage.

Figure 11. Contour map of damage index $I^{d}$ for square plate with side damage

Figure 12 Time-history diagram for the plate centre of the rectangular plate in the case of side defect. Harmonic loading with $\mathrm{P}=500 \mathrm{~N}, \omega_{\mathrm{e}}=260 \mathrm{rad} / \mathrm{s}$. Solid line - undamaged plate, Dotted line - Damaged plate. 
Figure 13. Poincaré map for undamaged and damaged in the plate centre of a rectangular plate with a side defect.

Figure 14. Contour map of damage index $I_{d}$ for rectangular plate with a side defectl

Figure 15 . Poincaré map for undamaged and damaged rectangular plate with a central defect.

Figure 16. Contour map of damage index $I_{d}$ for rectangular plate with a central defect. 